\title{
Flebite no pré e pós-operatório de pacientes neurocirúrgicos*
}

\author{
Phlebitis among neurosurgical patients
}

Flebitis en el pre y postoperatorio de pacientes neuroquirúrgicos

\section{Lucilene Reno Ferreira ${ }^{1}$, Mavilde da Luz Gonçalves Pedreira $^{2}$, Solange Diccini ${ }^{3}$}

\section{RESUMO}

Objetivos: Verificar a incidência de flebite em pacientes internados em uma unidade de neurocirurgia, o tempo de permanência de cateteres intravenosos periféricos e os possíveis fatores de risco para o desenvolvimento de flebite. Métodos: Estudo do tipo coorte prospectivo, com uma amostra 60 pacientes, nos quais foram inseridos 152 cateteres intravenosos periféricos do tipo sobre agulha. Resultados: Os resultados evidenciaram incidência de flebite de 10,5\%, em relação à proporcionalidade de cateteres. O tempo de permanência dos cateteres variou de 2 a 216 horas, sendo que os cateteres com permanência inferior a 72 horas tiveram menor incidência de flebite. Conclusões: Esta incidência encontra-se acima da aceita em padrões de prática internacionais. Os fatores de risco para flebite identificados neste estudo foram: tempo de permanência maior que 72 horas, manutenção intermitente dos cateteres e inserção no período de pós-operatório.

Descritores: Flebite; Período pós-operatório; Doenças do sistema nervoso/enfermagem; Infusões endovenosas

\begin{abstract}
Objectives: To identify the risk factors for the development of phlebitis, the length of time with a peripheral intravenous catheter, and the incidence of phlebitis among neurosurgical patients. Methods: this was a prospective cohort study. The sample consisted of 60 patients who underwent neurosurgery in a Brazilian hospital. A total of 152 peripheral intravenous catheters were identified. Results: The risk factors for the development of phlebitis were the permanence of catheter longer than 72 hours, intermittent maintenance of the catheters, and catheters that had been inserted in the postoperative period. The length of time with a catheter ranged from 2 to 216 hours. Phlebitis developed among $10.5 \%$ of patients. Conclusions: The incidence of phlebitis among this study's sample is higher than the rate recommended by the International Standards of Practice.
\end{abstract}

Descriptors: Phlebitis; Postoperative period; Nervous system diseases/nursing; Infusions, intravenous

\section{RESUMEN}

Objetivos: Verificar la incidencia de flebitis en pacientes internados en una unidad de neurocirugía, el tiempo de permanencia de catéteres intravenosos periféricos y los posibles factores de riesgo para el desarrollo de flebitis. Métodos: Estudio de tipo cohorte prospectivo, con una muestra de 60 pacientes, en los cuales fueron insertados 152 catéteres intravenosos periféricos de tipo sobre aguja. Resultados: Los resultados evidenciaron incidencia de flebitis en un 10,5\%, en relación a la proporción de catéteres. El tiempo de permanencia de los catéteres varió de 2 a 216 horas, siendo que los catéteres con permanencia inferior a 72 horas tuvieron menor incidencia de flebitis. Conclusiones: Esta incidencia se encuentra sobre la preconizada en patrones de práctica internacionales. Los factores de riesgo para flebitis identificados en este estudio fueron: tiempo de permanencia mayor a 72 horas, manutención intermitente de los catéteres e inserción en el período postoperatorio. Descriptores: Flebitis; Periodo postoperatorio; Enfermedades del sistema nervioso/enfermería; Infusiones Intravenosas

\footnotetext{
* O estudo foi desenvolvido com apoio do Programa de Iniciação Científica (PIBIC/CNPq na Universidade Federal de São Paulo - UNIFESP-São Paulo (SP), Brasil, em 2003/2004.

${ }^{1}$ Enfermeira do Programa da Saúde da Família - Fundação Zerbini, Unidade Básica de Saúde - São Paulo (SP), Brasil.

${ }^{2}$ Professora Adjunto do Departamento de Enfermagem da Universidade Federal de São Paulo - UNIFESP. São Paulo (SP), Brasil.

${ }^{3}$ Professora Adjunto do Departamento de Enfermagem da Universidade Federal de São Paulo UNIFESP- São Paulo (SP), Brasil.
} 


\section{INTRODUÇÃO}

A terapia intravenosa (TIV) evoluiu com o desenvolvimento de novas tecnologias, tornando-se indispensável na área da saúde. A instalação de um cateter intravenoso (IV) periférico para administração de terapia parenteral é um dos procedimentos invasivos mais freqüentes realizados em hospitais ${ }^{(1)}$. Somente nos Estados Unidos da América (EUA), estima-se que ocorram anualmente mais de 150 milhões de infusões intravasculares em 30 milhões de pacientes ${ }^{(2)}$. A administração de líquidos IV objetiva repor volume intravascular, corrigir déficits de eletrolítos, administrar fármacos, realizar hemodiálise e fornecer nutrientes ao paciente quando não há outra via disponível.

Porém, apesar da TIV relacionar-se com inúmeros benefícios terapêuticos, apresenta algumas desvantagens, pois a obtenção do acesso vascular, através da inserção de um cateter IV, não é um procedimento inócuo. A natureza invasiva deste tipo de procedimento tem preocupado profissionais de saúde responsáveis pela implementação da TIV, como enfermeiros e médicos. Infecções relacionadas a cateteres intravasculares são complicações que podem comprometer a saúde do paciente, bem como aumentar o tempo de internação hospitalar e gerar acréscimo nos custos de atendimento ${ }^{(3)}$.

O fato de $90 \%$ dos pacientes hospitalizados receberem soluções e medicamentos IV, coloca-os em risco de desenvolver complicações associadas, que podem ser identificadas em $50 \%$ a $75 \%$ dos pacientes que recebem TIV $^{(4-5)}$. Complicações locais relacionadas ao uso de cateteres IV ocorrem como reações adversas ou traumas, que se localizam ao redor do local da punção, sendo reconhecidas precocemente por avaliação objetiva. As principais complicações locais são: hematoma subcutâneo, ruptura da veia, obliteração do vaso, trombose, flebite, tromboflebite, infiltração, extravasamento e infecção local ${ }^{(4)}$.

Dos pacientes em TIV, 27\% a 70\% podem desenvolver algum estágio de flebite, fazendo com que essa complicação local seja uma das mais comuns da prática atual. É associada principalmente a cateteres sobre agulha, além de ser a causa mais comum de necessidade de remoção de cateteres intravenosos periféricos (CIP) ${ }^{(6-8)}$.

A flebite é definida como uma inflamação aguda de uma veia caracterizada por edema, dor, desconforto e eritema ao redor do local de inserção do CIP ou ao longo do trajeto da veia, sendo possível a evolução para um "cordão" venoso palpável. Usualmente, a dor e o edema local podem persistir por vários dias ou até por semanas. Existem diversos fatores que podem influenciar o desenvolvimento da flebite como: técnica inadequada de inserção do CIP, condição clínica do paciente, características da veia, incompatibilidade entre fármacos, tonicidade e pH do medicamento ou solução, filtração inefetiva, calibre, tamanho, comprimento e material do cateter, e tempo prolongado de inserção ${ }^{(9)}$.

Segundo a Intravenous Nurses Society a taxa aceitável de flebite em uma dada população de pacientes deve ser $5 \%$ ou menos. A enfermeira deverá ter competência e habilidade para avaliar o sítio de inserção do cateter e determinar a necessidade de tratamento e ou intervenção na ocorrência de flebite, devendo sempre ser documentada, usando-se um padrão ou uma escala de medida que irá pontuar os graus e a severidade da mesma $^{(10-11)}$

Atualmente, com o rápido avanço tecnológico relacionado a TIV, surge a necessidade de equipes e profissionais, principalmente de enfermeiros especialistas em TIV, promoverem o desenvolvimento de práticas fundamentadas em conhecimento científico ${ }^{(11-13)}$.

$\mathrm{Na}$ prática diária de cuidados de pacientes neurocirúrgicos, observamos que a TIV é freqüentemente utilizada durante o pré e pós-operatório. Durante a revisão da literatura, não encontramos pesquisas direcionadas a TIV e o paciente neurocirúrgico, o que nos motivou a realização deste estudo. Esta pesquisa teve como objetivo verificar a incidência de flebite em pacientes internados em uma unidade de neurocirurgia, comparando os resultados com o padrão de incidência (menor que $5 \%$ ) aceito pela Intravenous Nurses Society ${ }^{(10-11)}$, como também, identificar o tempo de permanência dos CIP e os possíveis fatores de risco para o desenvolvimento de flebite.

\section{MÉTODOS}

Estudo do tipo coorte prospectivo realizado na unidade de neurocirurgia de um hospital universitário da cidade de São Paulo, no período de outubro de 2003 a abril de 2004. O estudo foi aprovado pelo Comitê de Ética em Pesquisa da instituição, e cada paciente incluído na amostra, assinou o Termo de Consentimento Livre e Esclarecido.

Os pacientes incluídos no estudo eram adultos, com idade igual ou superior a 18 anos, de ambos os sexos, internados para cirurgia eletiva. Pacientes com quadro de agitação motora, confusão mental, com flebite prévia à internação, com CIP inseridos em situação de emergência ou internados na unidade com CIP, foram excluídos do estudo.

A avaliação inicial dos participantes foi realizada a partir da primeira inserção de CIP. Foram medidas as seguintes variáveis: sexo, idade, diagnóstico médico, cor da pele, total de CIP inseridos, tipo do cateter (cateter sobre agulha, cateter agulhado), tempo de internação hospitalar (total, pré-operatório, pós-operatório), inserção do CIP e período operatório, pacientes com flebite, relação CIP e 
incidência de flebite, grau de flebite, tempo de permanência do CIP, material do cateter (teflon ${ }^{\circledR}$ ou poliuretano), calibre do cateter (Gauge-G), profissional que realizou a punção venosa (enfermeiro(a), auxiliar/ técnico de enfermagem e outros) e local da punção venosa, fármacos e soluções administradas e causas da remoção do CIP. Também foram avaliadas as formas de manutenção do CIP (contínua, intermitente ou ambas). Caracteriza-se como forma de manutenção do CIP contínua, a administração de soluções e fármacos em intervalos de tempo superiores a duas horas e a forma de manutenção do CIP, intermitente, quando realizada a intervalos de tempo inferiores a duas horas ${ }^{(14)}$.

A flebite foi classificada conforme a intensidade em quatro graus $^{(10-11)}$ : Grau 1 - eritema com ou sem dor local ou edema, sem endurecimento e cordão fibroso não palpável; Grau 2 - mesmos sintomas da flebite de Grau 1, porém com endurecimento local; Grau 3 - além dos sinais clínicos do Grau 2, acrescenta-se a presença de um cordão fibroso palpável ao longo da veia; Grau 4 adicionalmente ao Grau 3, apresenta um cordão venoso palpável maior que 1 centímetro, com drenagem purulenta.

Os motivos de retirada do cateter foram categorizados em: alta do tratamento (final da TIV por alta hospitalar, término do esquema terapêutico ou óbito) e ocorrências adversas (infiltração, obstrução, dobra ou remoção acidental do cateter). Ocorrência adversa foi definida como qualquer evento indesejável que impossibilitou a continuidade do uso do CIP, podendo ser ou não conseqüência de falha dos profissionais no cuidado com os cateteres $^{(14)}$.

A avaliação do paciente foi realizada diariamente até a interrupção da TIV ou alta do paciente. O sítio de inserção e a região ao redor eram observados visualmente e por palpação para investigar a possível presença de sinais inflamatórios. A partir do diagnóstico da flebite, o cateter era retirado e nova veia era puncionada de acordo com a necessidade. Manteve-se a padronização proposta pela instituição de estudo quanto a técnica de punção venosa, com o emprego de álcool a $70 \%$ para a anti-sepsia da pele, e a fixação dos cateteres com fita adesiva impermeável, tipo esparadrapo.

Os dados não-paramétricos foram analisados usando o teste de Qui-quadrado ou teste exato de Fisher. Neste teste fixou-se em 5\% ( $\mathrm{p} \leq 05)$ o nível para rejeição da hipótese de nulidade, assinalando-se com asteriscos os valores significantes.

\section{RESULTADOS}

Neste estudo, foram incluídos 60 pacientes, os quais foram submetidos a inserção de 152 CIP do tipo cateter sobre agulha. Dos 60 pacientes, 31 (51,7\%) eram do sexo feminino e $29(48,3 \%)$ do sexo masculino, com uma mediana de idade de 46 anos, variando de 18 a 84 anos. A cor da pele predominante foi branca em $37(61,6 \%)$ pacientes, cor parda em $16(26,7 \%)$, preta em seis $(10,0 \%)$ e amarela em um $(1,7 \%)$ paciente. Os diagnósticos médicos foram categorizados em quatro grupos, sendo que $36(60,0 \%)$ pacientes eram portadores de tumores intracranianos ou medulares, nove $(15,0 \%)$ de lesões de nervos periféricos, oito $(13,3 \%)$ com patologias cerebrovasculares (aneurismas e malformações arteriovenosas) e sete $(11,7 \%)$ patologias medulares (hérnias discais e estenose do canal raquidiano). A mediana do tempo de internação hospitalar foi de 10,5 dias, variando de 1 a 53 dias. No período pré-operatório a mediana foi de quatro dias, variando de 1 a 33 dias. No período pós-operatório a mediana foi de seis dias variando de 1 a 42 dias. Dos 152 CIP analisados, 58 $(38,1 \%)$ foram inseridos no pré-operatório, 58 (38,1\%) no pós-operatório e $36(23,8 \%)$ no trans-operatório.

Dos 60 pacientes, $11(18,3 \%)$ evoluíram com flebite, sendo que oito $(72,7 \%)$ eram do sexo masculino e três $(27,3 \%)$ do feminino. Quando analisada a incidência de flebite entre os 152 cateteres periféricos, ocorreram 16 $(10,5 \%)$ episódios de flebite, distribuídos da seguinte maneira: nove pacientes tiveram apenas um episódio de flebite, um paciente teve três episódios de flebite e um teve quatro episódios de flebite. Dos 16 episódios de flebite, 11 ocorreram em homens e cinco ocorreram em mulheres, não havendo relação estatisticamente significante entre sexo e flebite $(\mathrm{p}=0,14)$. Segundo a cor da pele, dos 16 casos de flebite, 10 ocorreram em pacientes da cor branca, cinco em pacientes da cor preta e um em paciente da cor parda, não havendo relação estatisticamente significante entre cor da pele e flebite $(p=0,07)$. Das 16 flebites, 11 surgiram no período pósoperatório e em cateteres inseridos no pós-operatório, três no pós-operatório em cateteres inseridos no transoperatório e dois no pré-operatório em cateteres inseridos no pré-operatório, com uma relação estatisticamente significante $(\mathrm{p}=0,02)$.

Considerando os $152 \mathrm{CIPs}$, observamos que 123 $(80,9 \%)$ foram instalados em pacientes com 60 anos ou menos e 29 (19,1\%) em pacientes com mais de 60 anos. Dos 16 episódios de flebite, 13 ocorreram em pacientes com idade de 60 anos ou menos e três em pacientes com mais de 60 anos. Não houve relação estatisticamente significante entre os cateteres inseridos em pacientes com mais ou menos de 60 anos e a presença de flebite $(p=0,97)$.

Quando avaliado o grau de flebite, oito foram Grau 1, sete Grau 2 e um foi Grau 3, nenhuma das flebites evoluiu para Grau 4.

A mediana de tempo de permanência do cateter foi de 24 horas, variando de 2 a 216 horas. A Tabela 1 
mostra a distribuição dos episódios de flebite segundo o tempo de permanência dos cateteres.

Tabela 1-Ocorrência de flebite em pacientes no período pré e pós-operatório de tratamento neurocirúrgico, segundo tempo de permanência dos cateteres.

\begin{tabular}{lccccccc}
\hline $\begin{array}{c}\text { Tempo de } \\
\begin{array}{c}\text { Permanência } \\
\text { dos cateteres }\end{array}\end{array}$ & Sem flebite & \multicolumn{2}{c}{$\begin{array}{c}\text { Com } \\
\text { flebite }\end{array}$} & \multicolumn{2}{c}{ Total } \\
\hline & $\mathrm{n}$ & $\%$ & $\mathrm{n}$ & $\%$ & $\mathrm{n}$ & $\%$ \\
$<72$ horas & 109 & 80,1 & 6 & 37,5 & 115 & 75,7 \\
$\geq 72$ horas & 27 & 19,9 & 10 & 62,5 & 37 & 24,3 \\
\hline Total & 136 & 100,0 & 16 & 100,0 & 152 & 100,0 \\
\hline
\end{tabular}

Teste de Qui-quadrado $\quad \mathrm{p}=0,0006^{*}$

Dos 115 (75,7\%) CIP que permaneceram no local de inserção por até 72 horas, $78(67,8 \%)$ permaneceram no local por 24 horas ou menos. Considerando isoladamente os cateteres que permaneceram no local de inserção por 72 horas ou mais, houve uma taxa de flebite de $27,0 \%$ e se analisarmos apenas os CIP inseridos por menos de 72 horas, a taxa de flebite foi de 5,2\%. Nos CIP que permaneceram por menos de 72 horas, ocorreram menos casos de flebite. Houve relação estatisticamente significante entre o tempo de permanência do cateter e a ocorrência de flebite $\left(\mathrm{p}=0,0006^{*}\right)$.

Do total de cateteres analisados, 130 (85,5\%) eram de teflon ${ }^{\circledR}$ e $22(14,5 \%)$ de poliuretano. Dentre os CIP de teflon ${ }^{\circledR}$, em $15(11,5 \%)$ ocorreu flebite, e dentre os de poliuretano, em um $(4,5 \%)$ cateter ocorreu flebite. Não houve relação estatisticamente significante entre o material dos cateteres e a ocorrência de flebite $(\mathrm{p}=0,54)$.

$\mathrm{Na}$ Tabela 2 é demonstrada a relação entre flebite e calibre do cateter.

Tabela 2 - Ocorrência de flebite em pacientes no período pré e pós-operatório de tratamento neurocirúrgico, segundo o calibre do cateter intravenoso periférico.

\begin{tabular}{lrrrrrrr}
\hline \multirow{2}{*}{ Calibre do cateter } & \multicolumn{2}{c}{ Sem flebite } & \multicolumn{2}{c}{$\begin{array}{c}\text { Com } \\
\text { flebite }\end{array}$} & \multicolumn{2}{c}{ Total } \\
\cline { 2 - 8 } & \multicolumn{1}{c}{$\mathrm{n}$} & \multicolumn{1}{c}{$\%$} & $\mathrm{n}$ & $\%$ & \multicolumn{1}{c}{$\mathrm{n}$} & \multicolumn{1}{c}{$\%$} \\
\hline 14 Gauge & 7 & 5,1 & 1 & 6,2 & 8 & 5,2 \\
16 Gauge & 10 & 7,4 & 1 & 6,3 & 11 & 7,2 \\
18 Gauge & 30 & 22,0 & 0 & 0,0 & 30 & 19,7 \\
20 Gauge & 49 & 36,0 & 8 & 50,0 & 57 & 37,6 \\
22 Gauge & 38 & 28,0 & 6 & 37,5 & 44 & 29,0 \\
24 Gauge & 2 & 1,5 & 0 & 0,0 & 2 & 1,3 \\
\hline Total & 136 & 100,0 & 16 & 100,0 & 152 & 100,0 \\
\hline
\end{tabular}

Teste de Qui-quadrado $\mathrm{p}=0,35$
A maioria das punções foi realizada com cateteres de menor calibre, de $20 \mathrm{G}(37,6 \%)$ e $22 \mathrm{G}(29,0 \%)$. Dentre os episódios de flebite, oito $(50,0 \%)$ ocorreram com cateteres de calibre 20G. Considerando os grupos de cateteres separadamente, a ocorrência de flebites foi semelhante entre os grupos de 14G, 20G e 22G. Não houve relação significante, considerando o calibre dos cateteres utilizados e a ocorrência de flebite $(\mathrm{p}=0,35)$.

A Tabela 3 mostra que os cateteres foram predominantemente inseridos na veia cefálica $(37,5 \%)$ e na veia basílica $(21,7 \%)$.

Tabela 3 - Ocorrência de flebite em pacientes no período pré e pós-operatório de tratamento neurocirúrgico segundo vasos de inserção do cateter intravenoso periférico.

\begin{tabular}{|c|c|c|c|c|c|c|}
\hline \multirow[t]{2}{*}{ Vasos de inserção } & \multicolumn{2}{|c|}{$\begin{array}{l}\text { Sem } \\
\text { flebite }\end{array}$} & \multicolumn{2}{|c|}{$\begin{array}{c}\text { Com } \\
\text { flebite }\end{array}$} & \multicolumn{2}{|c|}{ Total } \\
\hline & $\mathrm{n}$ & $\%$ & $\mathrm{n}$ & $\%$ & $\mathrm{n}$ & $\%$ \\
\hline Arco dorsal da mão & 12 & 8,8 & 1 & 6,2 & 13 & 8,6 \\
\hline Cefálica & 51 & 37,5 & 6 & 37,5 & 57 & 37,5 \\
\hline Cefálica acessória & 20 & 14,7 & 2 & 12,5 & 22 & 14,5 \\
\hline Basílica & 28 & 20,6 & 5 & 31,3 & 33 & 21,7 \\
\hline Mediana & 4 & 2,9 & 0 & 0 & 4 & 2,6 \\
\hline Mediana cubital & 21 & 15,5 & 2 & 12,5 & 23 & 15,1 \\
\hline Total & 136 & 100,0 & 16 & 100,0 & 152 & 100,0 \\
\hline
\end{tabular}

Teste de Qui-quadrado $\mathrm{p}=0,98$

As flebites ocorreram predominantemente na veia cefálica. Dos episódios de flebite, nenhuma ocorreu em veias do braço. Não houve relação estatisticamente significante entre as veias utilizadas para os CIP e a ocorrência de flebites.

A Tabela 4 mostra que forma de manutenção do cateter de maior freqüência foi contínua $(48,7 \%)$. As flebites ocorreram predominantemente em CIP mantidos de modo intermitente $(75,0 \%)$. Houve relação estatisticamente significante entre a ocorrência de flebites e a forma de manutenção dos cateteres intravenosos periféricos.

Quando avaliado o profissional que executou a punção venosa dos 152 cateteres, 41 (26,9\%) foram realizados por enfermeiros, sendo que seis $(14,6 \%)$ evoluíram com flebite, $72(47,4 \%)$ foram realizados por auxiliares ou técnicos de enfermagem, sendo que sete $(9,7 \%)$ evoluíram com flebite e $39(25,7 \%)$ foram puncionados por outros profissionais (anestesistas, alunos de graduação em enfermagem) sendo que três $(7,7 \%)$ evoluíram com flebite $(\mathrm{p}=0,57)$.

A análise da ocorrência de flebite segundo os tipos de fármacos e ou soluções administrados evidenciou correlação sem significância estatística $(p=0,98)$. 
Tabela 4 - Ocorrência de flebite em pacientes no período pré e pós-operatório de neurocirurgia, segundo a forma de manutenção dos cateteres intravenosos periféricos.

\begin{tabular}{lccccccc}
\hline $\begin{array}{c}\text { Manutenção } \\
\text { do cateter }\end{array}$ & \multicolumn{2}{c}{ Sem flebite } & \multicolumn{2}{c}{$\begin{array}{c}\text { Com } \\
\text { flebite }\end{array}$} & \multicolumn{2}{c}{ Total } \\
\cline { 2 - 8 } & $\mathbf{n}$ & $\mathbf{0}$ & $\mathbf{n}$ & $\mathbf{0}$ & $\mathbf{n}$ & $\mathbf{\%}$ \\
\hline Contínua & 72 & 52,9 & 2 & 12,5 & 74 & 48,7 \\
Intermitente & 43 & 31,6 & 12 & 75,0 & 55 & 36,2 \\
Ambas & 21 & 15,5 & 2 & 12,5 & 23 & 15,1 \\
\hline Total & 136 & 100,0 & 16 & 100,0 & 152 & 100,0 \\
\hline
\end{tabular}

Teste de Qui-quadrado $\mathrm{p}=0,002^{*}$

Destacaram-se soros de manutenção com eletrólitos $(21,2 \%)$, antibióticos $(15,5 \%)$, analgésicos $(15,2 \%)$, antiinflamatórios $(14,8 \%)$, antiácidos $(12,0 \%)$, antieméticos $(8,9 \%)$, anticonvulsivantes $(8,8 \%)$, anti-hipertensivos $(1,4 \%)$, diuréticos $(1,1 \%)$, anticoagulantes $(0,7 \%)$, e glicose a $50 \%$ em água $(0,4 \%)$. Todos os pacientes receberam mais de um grupo de drogas durante a terapia intravenosa periférica.

Dos 152 cateteres, 51 (33,5\%) foram retirados devido à alta do tratamento, sendo $38(25,0 \%)$ por término da TIV e $13(8,5 \%)$ por alta hospitalar. Dos 152 cateteres, $57(37,5 \%)$ foram retirados devido a ocorrências adversas, sendo $22(14,4 \%)$ por obstrução do cateter, $16(10,5 \%)$ por flebite, $12(8,0 \%)$ por infiltração, seis $(4,0 \%)$ por remoção acidental do cateter e um $(0,6 \%)$ por dobra do cateter. Dos $44(29,0 \%)$ CIP restantes, 38 (25,0\%) foram retirados devido à presença de um cateter venoso central e seis $(4,0 \%)$ por outras ocorrências, referentes às punções em locais inadequados.

\section{DISCUSSÃO}

A taxa de flebite neste estudo foi de 10,5\%, superior ao padrão aceito de 5\%, considerando os episódios de flebite e o número total de cateteres inseridos. $\mathrm{Na}$ literatura, os estudos são pouco homogêneos com relação às taxas de incidência de flebites. A variação pode ser de $2 \%$ a 50\%, levando-se em conta que nos estudos há diferença com relação às amostras, seleção de pacientes, métodos, desenho e, também, devido ao uso não padronizado das definições e critérios diagnósticos para flebite $^{(15-16)}$.

Dentre os fatores de possível influência na ocorrência de flebite, está o sexo. Estudos demonstram tanto predomínio no sexo feminino, como no sexo masculino $(9,17)$. Neste estudo ocorreu maior freqüência de flebites em pacientes do sexo masculino $(68,7 \%)$, porém não sendo estatisticamente significante a diferença.

A cor da pele branca foi predominante entre os pacientes $(61,6 \%)$ deste estudo. Quando analisada a taxa de flebite e a cor não encontramos relação estatisticamente significante, porém na literatura há maior incidência de flebite na cor branca ${ }^{(4,14)}$.

A mediana do tempo de permanência dos cateteres periféricos foi de 24 horas. A recomendação do Center for Desease Control and Prevention (CDC) é que haja troca de CIP a cada 72 a 96 horas. Destaca-se que esta recomendação não é adotada pela unidade de neurocirurgia, sendo que os cateteres são retirados ou trocados na presença de complicações ou no final da TIV. Em crianças, há evidência científica de que não há necessidade da troca do cateter periférico intravenoso ${ }^{(12)}$. Em nosso estudo verificamos que o tempo de permanência maior ou igual a 72 horas foi um fator de risco para flebite $(p=0,0007)$. Encontramos que 37,5\% das flebites ocorreram nos primeiros três dias de permanência do cateter e após o $4^{\circ}$ dia, a taxa da flebite foi de $62,5 \%$. Na literatura há uma diversidade de dados, com relação ao tempo de permanência do cateter e a ocorrência de flebite, sendo que há maior incidência entre o $3^{\circ}$ e $4^{\circ}$ dia, tornando-se mais constante após o $5^{\circ}$ dia de permanência ${ }^{(1,15-18)}$.

As flebites ocorreram predominantemente em cateteres inseridos no trans e pós-operatório $(p=0,02)$. Para os pacientes com CIP no pré-operatório ressalta-se que a indicação da terapia intravenosa estava associada à hidratação, uso de drogas anti-inflamatórias e anticonvulsivantes. Diferentemente do pós-operatório, que além destas drogas, havia a associação de anestésicos, antibióticos e maior tempo de internação. Estudo realizado comparando cateteres utilizados nos períodos trans e pós-operatório mostrou maior incidência de flebite quando comparado com o grupo onde os cateteres eram inseridos exclusivamente no pós-operatório ${ }^{(1)}$. Verificamos que a flebite ocorreu em mais de uma vez nos mesmos pacientes, quando possuíam um maior número de cateteres inseridos, sendo que nestes pacientes os graus de flebite eram mais intensos, grau 2 e 3, como também foram aqueles que utilizaram por mais tempo antibioticoterapia. Dados da literatura mostram altas taxas de flebite no uso de antibioticoterapia prolongada ${ }^{(7,8,17)}$. Entretanto, não se verificou neste estudo relação entre fármacos e soluções administrados e ocorrência de flebite.

Dos cateteres inseridos, a maioria $(85,5 \%)$ era de teflon ${ }^{\circledR}$, sendo que a grande parte das flebites ocorreu em cateteres deste tipo de material, porém não houve diferença estatisticamente significante entre teflon ${ }^{\circledR}$ e poliuretano e ocorrência de flebite, diferentemente da literatura. Vários estudos já demonstraram que a incidência de flebite em cateteres de teflon ${ }^{\circledR}$ é maior que nos cateteres de poliuretano ${ }^{(4,15,18)}$.

Os cateteres mais inseridos tinham o calibre de $20 \mathrm{e}$ 22 gauge, considerados os de menor calibre para adultos, porém não houve diferença estatisticamente significante 
quando comparados os grupos com e sem flebite e o calibre do cateter. A literatura mostra que os cateteres de maior calibre (14 a 18 gauge) produzem maiores taxas de flebite, principalmente pelo fato de exercerem maior atrito contra a parede do vaso cateterizado ${ }^{(4,15,17-18)}$.

Em relação à forma de manutenção do cateter e flebite, não encontramos dados na literatura. Verificamos em nosso estudo que a forma contínua foi a mais utilizada, e a forma intermitente foi a que estatisticamente se relacionou a maior taxa de flebite $(p=0,002)$. Pode-se inferir que este resultado tem relação com a maior manipulação do cateter, podendo acarretar flebite mecânica.

As veias mais utilizadas para a inserção dos CIP foram a cefálica e a basílica. Não encontramos associação estatisticamente significante entre as veias utilizadas para inserção dos CIP e a ocorrência de flebite, apesar da veia cefálica ter apresentado a maior taxa de flebite $(37,5 \%)$. Verificamos que na literatura, as veias do antebraço e as da fossa antecubital apresentam maiores taxas de flebite do que as do dorso da mão e/ou pulso ${ }^{(4,18)}$.

As ocorrências adversas aconteceram em $37,5 \%$ das situações analisadas. As ocorrências adversas como motivo de retirada dos cateteres devem ser enfatizadas, na medida em que podem ser decorrentes de resultados de cuidado ineficaz, desde a inserção do CIP até o final da terapia intravenosa. Este cuidado é responsabilidade da enfermeira e da sua equipe, incluindo contínua atualização de conhecimentos em relação aos cuidados, bem como, prevenção das complicações da terapia intravenosa. A prevenção de complicações relacionadas ao uso de CIP, em locais que contam com equipe de TIV, demonstra que enfermeiras especialmente treinadas podem reduzir a incidência de complicações locais e sistêmicas relacionadas aos $\mathrm{CIP}^{(12,19,20)}$. Em pacientes que receberam os cuidados de enfermeiras da equipe de TIV, a taxa de complicações locais foi de $7,9 \%$ contra $21,7 \%$ das complicações encontradas na equipe não treinada ${ }^{(13)}$.

Segundo os padrões estabelecidos pela Intravenous Nurses Society, a enfermeira responsável pela terapia deve ter grande conhecimento teórico e habilidade prática, bem como conhecer as indicações, efeitos colaterais e possíveis reações adversas. Deve elaborar o plano de cuidados, acompanhar a evolução e a efetividade da terapia prescrita, documentando a resposta do paciente e as intervenções necessárias para se alcançar o resultado esperado ${ }^{(11)}$.

\section{CONCLUSÃO}

A terapia intravenosa é freqüentemente utilizada em unidades hospitalares. O paciente neurocirúrgico tanto na fase do pré como pós-operatório necessita da TIV durante o seu tratamento. Esta pesquisa concluiu que a incidência de flebite em relação aos cateteres inseridos em veias periféricas foi de 10,5\%, sendo o dobro da taxa aceita literatura. O tempo de permanência dos cateteres variou de 2 a 216 horas, sendo que os cateteres instalados em períodos inferiores a 72 horas tiveram a menor incidência de flebite. Os fatores de risco para flebite foram: tempo de permanência maior que 72 horas, manutenção intermitente dos cateteres e inserção no período de pósoperatório.

\section{REFERÊNCIA}

1. Panadero A, Iohom G, Taj J, Mackay N, Shorten G. A dedicated intravenous cannula for postoperative use effect on incidence and severity of phlebitis. Anaesthesia. 2002; 57(9): 921-5.

2. Pereira RCC, Zanetti ML. Complicações decorrentes da terapia intravenosa em pacientes cirúrgicos. Rev Latinoam Enfermagem. 2000; 8(5): 21-7.

3. Fernandes AT, Filho NR. Infecção do acesso vascular. In: Fernandes AT, Fernandes MOV, Ribeiro Filho N, Graziano KU, Gabrielloni MC, Cavalcante NJF, Lacerda RA (Editores). Infecção hospitalar e suas interfaces na área da saúde. São Paulo: Atheneu; 2000. p.556-79.

4. Phillips LD. Manual de terapia intravenosa. 2a ed. Porto Alegre: Artmed; 2001. p. 236-46.

5. Lundgren A, Wahren LK. Effect of education on evidencebased care and handling of peripheral intravenous lines. J Clin Nurs. 1999; 8(5): 577-85.

6. Chukhraev AM, Grekov IG, Aivazyan, M. Local complications of nursing interventions on peripheral veins. J Intraven Nurs. 2000; 23(3):167-9.

7. Monreal M, Quilez F, Rey-Joly C, Rodriguez S, Sopena N, Neira C, Roca J. Infusion phlebitis in patients with acute pneumonia: a prospective study. Chest. 1999; 115(6): 157680.

8. de Dios García-Diaz J, Santolaya Perrín R, Paz Martínez Ortega M, Moreno-Vázquez M. Flebitis relacionada con la administración intravenosa de antibióticos macrólidos. Estudio comparativo de eritromicina y claritromicina. Med Clin (Barc). 2001;116(4):133-5.

9. White SA. Peripheral intravenous therapy-related phlebitis rates in an adult population. J Intraven Nurs. 2001; 24(1):19-24.

10. Intravenous Nurses Society. Infusion nursing standards of practice. J Intraven Nurs. 2000. 23(6S):S1-S46.

11. Infusion Nurses Society. Infusion nursing standards of practice. J Infus Nurs. 2006. 29(1 Suppl): S1-92.

12. Associação Paulista de Estudos e Controle de Infecção Hospitalar. Infecção associada ao uso de cateteres vasculares. 3a ed. rev. São Paulo; 2005. p.1-100.

13. Soifer NE, Borzak S, Edlin BR, Weinstein RA. Prevention of peripheral venous catheter complications with an intravenous therapy team: a randomized controlled trial. Arch Intern Med. 1998; 158(5): 473-7.

14. Machado AF, Pedreira MLG, Chaud MN. Estudo prospectivo, randomizado e controlado sobre o tempo de permanência de cateteres venosos periféricos em crianças, segundo três tipos de curativos. Rev Latinoam Enfermagem. 2005; 13(3): 291-8.

15. Tagalakis V, Kahn SR, Libman M, Blostein M. The 
epidemiology of peripheral vein infusion thrombophlebitis: a critical review. Am J Med. 2002; 113(2):146-51.

16. Lanbeck P, Odenholt I, Paulsen O. Perception of risk factors for infusion phlebitis among Swedish nurses: a questionnaire study. J Infus Nurs. 2004; 27(1):25-30.

17. Lanbeck P, Odenholt I, Paulsen O. Antibiotics differ in their tendency to cause infusion phlebitis: a prospective observational study. Scand J Infect Dis. 2002; 34(7): 512-9.

18. Karadag A, Gorgulu S. Effect of two different short peripheral catheter materials on phlebitis development. J Intraven Nurs. 2000; 23(3):158-66.

19. Monreal M, Oller B, Rodriguez N, Vega J, Torres T, Valero P, et al. Infusion phlebitis in post-operative patients: when and why. Haemostasis. 1999; 29(5):247-54.

20. Jacobson AF, Winslow EH. Variables influencing intravenous catheter insertion difficulty and failure: an analysis of 339 intravenous catheter insertions. Heart Lung. 2005; 34(5): 345-59. 